\title{
Comparative Study of Cleansing Action of Some Detergents Available in Nepalese Market
}

\author{
Arvind Pathak ${ }^{1 *}$, Pratima Khadka ${ }^{1}$ and Prabin Karki ${ }^{1}$ \\ ${ }^{1}$ Department of Chemistry, Tri-Chandra Multiple Campus, Tribhuvan University, Kathmandu
}

\author{
*CORRESPONDENCE: \\ Arvind Pathak \\ Department of Chemistry, Tri-Chandra \\ Multiple Campus, Tribhuvan University, \\ Kathmandu \\ Email: apathak2070@gmail.com
}

ISSN : 2382-5359(Online), 1994-1412(Print)

DOI:

https://doi.org/10.3126/njst.v20i1.39435

\section{ACCESS THE ARTICLE ONLINE}

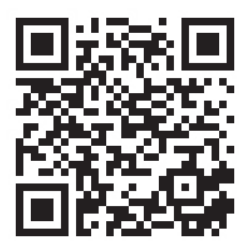

\section{CONFILICT OF INTEREST: None}

Copyright: The Author(s) 2020. This is an open access article under the $\underline{\mathrm{CC} \text { BY license. }}$

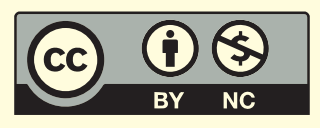

\begin{abstract}
Detergents commercially available in the Nepalese market were studied and several parameters such as surface tension, $\mathrm{pH}$, critical micelle concentration, foaming stability test, hard water test, emulsions stability test were performed. Different medium such as ground water (G.W.), tap water (T.W.), distilled water (D.W.) and 5\% ethanol in distilled water were selected for this study. The decrease in surface tension and critical micelle concentration (CMC) in ground water, tap water, distilled water and 5\% ethanol in distilled water, ease of cleansing action of the detergents in this medium have been found of the following order: $5 \%$ ethanol in distilled water $>$ distilled water (D.W.) $>$ tap water (T.W.) > ground water (G.W.). Among the four detergents, the D1 have shown the least surface tension, $\mathrm{CMC}$ value, foam collapsing time, the weight of scum formed when treated with hard water. And maximum emulsion stability of the detergent D1 determines good quality detergent.
\end{abstract}

Keywords: Critical micelles concentration (CMC), emulsion, foaming, scum, surface tension.

\section{INTRODUCTION}

In recent years, synthetic soapless detergents are being commonly used for laundering, dish-washing and cleaning. In all cases, the cleansing action is based upon the same principle for replacing the undesired dirt with a detergent, leaving the dirt in a suspended and protected state so that it is easily removed by running water (Khetrapal et al. 2015). The first synthetic detergent was developed in Germany during World war-I. Synthetic detergents are cleaning products prepared chemically from a variety of raw materials. The first detergent was mainly used for hand dish-washing and adequate fabric laundering. As a detergent, the surfactant is a basic essential cleaning ingredient, while the builder such as sodium tripolyphosphate softens water by holding calcium and magnesium ions present in water and prevents to react with the ingredients of the detergent and vastly improves the cleansing action. In response to a shortage of animal and vegetable fats and oils during World War I and World War II, detergent surfactants were developed. It increases the effectiveness of cleaning action because it contains one or more surfactants (www.cleaning101.com). 
When the micelle formation completes then the micelle of soap becomes detectable. The concentration of the surfactant above which micelles form is called critical micelle concentration (CMC). The increase of soap concentration up to critical micelle concentration decreases the surface tension (Cutler 1972). Micellar aggregates formation causes significant changes in the surfactant physical properties, such as conductivity and surface tension, which sharply changes near the CMC forms' concentration (Chakraborty et al. 2011; Elarbi et al. 2020).

The tendency to form micelle in solution largely depends on the nature and type of the surfactant. Surfactants with longer hydrophobic tail have a greater tendency of micelles formation. The more robust, hydrophobic effect decreases $\mathrm{CMC}$, and thus larger micelles are formed (Bratovcic et al. 2018). Three different phases have been identified from the surfactants' surface tension value at different concentrations (Khan \& Shah 2008).

a. At very low surfactant concentration surface tension of the solution remains almost constant.

b. Addition of surfactant decreases the surface tension drastically.

c. At CMC point, surface becomes saturated and surfactant molecules' Addition does not affect the surface tension.

Pisarcik et al. found the linear dependence of the aggregation number on surfactant concentration. The increase in aggregation number on surfactant significantly increases the micellar growth (Pisarcik et al. 2015). The decrease in CMC values of sodium dodecylsulphate solution with the Addition of $\mathrm{Na}_{2} \mathrm{SO}_{4}$ has been studied (Limbu et al. 2014).

The addition of detergents in water reduces the surface tension of water and also reduces the interfacial tension between water and dirt particles and therefore dirt particles coalesce forming emulsion. Good quality detergents usually form less scum in hard water; addition of a suitable builder improves the cleaning efficiency of the detergents.

With the increasing demand for soap and detergents, different brands of these cleansing agents are available in the Nepalese market. A study on the quality of these cleansing agents in different aqueous mediums seems needed to provide a guideline for selecting detergent and aqueous medium to save time, money and water consumption.
This study aimed to study physicochemical properties of commercially available detergents in the Nepalese market in different sources of aqueous medium and correlate their cleansing action.

\section{MATERIALS AND METHODS}

\subsection{Materials}

Four commonly used detergents available in the local market were selected to study their cleansing action in different medium such as ground water (G.W.), tap water (T.W.), distilled water (D.W.) and 5\% ethanol in distilled water. Apparatus such as Beaker, Measuring Flask, stalagmometer and specific gravity bottle, $\mathrm{pH}$ meter (Hanna instruments) and chemicals such as ethanol, $\mathrm{MgCl}_{2} \cdot 6 \mathrm{H}_{2} \mathrm{O}$ (97\%, Thermo Fisher Scientific, India), $\mathrm{FeCl}_{3}$ (96\%, Thermo Fisher Scientific, India), and $\mathrm{CaCl}_{2} \cdot 2 \mathrm{H}_{2} \mathrm{O}$ (98\%, Thermo Fisher Scientific, India) were used in this study.

\subsection{Sample Preparation}

The solution of detergents were prepared and used to measure surface tension $(0.1 \%), \mathrm{pH}(0.1 \%), \mathrm{CMC}(0.01$ to $0.1 \%$ ), foaming stability $(0.1 \%)$, hardness $(2 \%)$, emulsions stability (1\%) of the detergents D1, D2, D3, D4, in 5\% ethanol in distilled water, distilled water, tap water and ground water (Khetrapal et al. 2015). And detergents solutions were prepared and several parameters such as surface tension, $\mathrm{pH}$, critical micelle concentration foam stability, hardness of water, and emulsion stability test were considered for study to estimate the detergents' cleansing action.

\subsection{Physical Properties of Detergent Solutions}

\subsubsection{Surface Tension}

Number of drops for the same volume of each detergent solution, distilled water, and weight were measured using stalagmometer and specific gravity bottle respectively. The surface tension of the detergents solution and distilled water was measured using the formula (Saha et al. 2011; Khetrapal et al. 2015).

$$
\begin{aligned}
& \gamma_{2}=\left[\left(\frac{\mathrm{n}_{1}}{\mathrm{n}_{2}}\right) \times\left(\frac{\mathrm{d}_{2}}{\mathrm{~d}_{1}}\right)\right] \times \gamma_{1} \\
& \text { Where, } \gamma_{1}, n_{1} d_{1} \text { and } \gamma_{2}, n_{2}, d_{2}
\end{aligned}
$$

where, are the surface tensions, number of drops and densities of water and detergent solution respectively. 


\subsection{2 pH of the Detergent Solutions}

At first, the $\mathrm{pH}$ metre was calibrated using buffer solutions of $\mathrm{pH} 4.0$ and 9.2. Then the solution's $\mathrm{pH}$ was measured at room temperature $\left(25^{\circ} \mathrm{C}\right)$ by using a $\mathrm{pH}$ meter.

\subsubsection{Critical Micelle Concentration (CMC)}

Detergent form associative colloids or micelles in an aqueous solution that decreases water's surface tension. As the surface tension decreases, the extent of adsorption of dirt in micelles increases and the detergent's cleansing action increases. Solutions of ten different concentrations of the detergents D1, D2, D3, and D4 were prepared and used to measure the detergents solution's surface tension and distilled water.

\subsubsection{Foaming Stability Test}

Foam stability tests were performed using $10 \mathrm{~mL}$ of detergent solutions separately in test tubes and were shaken for 10 times. The time for disappearance of $2 \mathrm{~mm}$ width of foam was recorded (Khetrapal et al. 2015).

\subsubsection{Hard Water Test}

For the hard water test, 2\% detergent solutions were prepared by the dissolution of the detergent followed by filtration. $15 \mathrm{~mL}$ of each detergent solution were taken in separate test tubes. Then 10 drops of $5 \% \mathrm{MgCl}_{2}, 5 \% \mathrm{FeCl}_{3}$, and $5 \% \mathrm{CaCl}_{2}$ solutions were added individually. The precipitate of the solutions was filtered, dried and weight (Khetrapal et al. 2015).

\subsubsection{Emulsion Stability Test}

For the emulsion stability test, $1 \%$ detergent solutions were prepared. This test was performed by taking $5 \mathrm{~mL}$ of detergent solutions; $0.5 \mathrm{~mL}$ of mustard oil and petrol were added separately and shaken for 1 minute. The time was recorded when the solution became clear (Khetrapal et al. 2015).

\section{RESULTS AND DISCUSSION}

\subsection{Surface Tension}

The surface tension values of the detergents D1, D2, D3 and D4 in different medium, i.e., ground water (G.W.), tap water (T.W.), distilled water (D.W.) and 5\% ethanol in distilled water, were found to be of the following order: D1
$<\mathrm{D} 2<\mathrm{D} 3<\mathrm{D} 4$ and are shown in the following Figure 1:

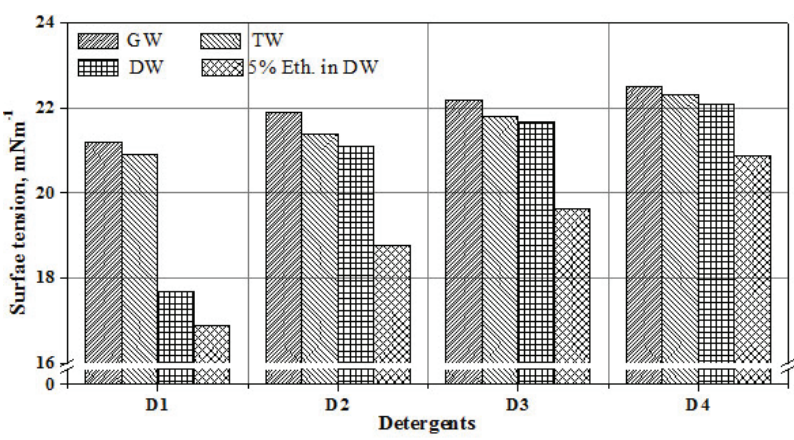

Fig. 1. Surface tension of $0.1 \% \mathrm{w} / \mathrm{v}$ of the detergent solution in a different medium

The decrease in surface tension is significant as the extent of decreased surface tension determines the effectiveness of a detergent's cleansing action. Therefore the cleansing action of the detergents are of the following order: D1 > D2 $>$ D3 $>$ D4.

The order of cleansing action in the medium varies as: $5 \%$ ethanol in distilled water $>$ distilled water (D.W.) $>$ tap water (T.W.) $>$ ground water (G.W.) shown in the Figure 2. This may be due to the fact that $5 \%$ ethanol in distilled water may dissolve the more hydrophobic part of the detergents and increase the aggregation number due to ethanol's less polar nature. Also, water and 5\% ethanol's surface tension in water is 72.75 and $56.41 \mathrm{mNm}$ ${ }^{1}$ respectively, at $20{ }^{\circ} \mathrm{C}$ (Vazquez et al. 1995). The lower value of surface tension improves the cleansing action of the detergent (Hazra 2015). Whereas in tap water and ground water impurities such as hardness and iron content may decrease the detergent's cleansing action. Addition of ethanol in water is expected to reduce the surface tension of the medium and also increase the solubilization capacity of the surfactant solution (Taylor et al. 2004).

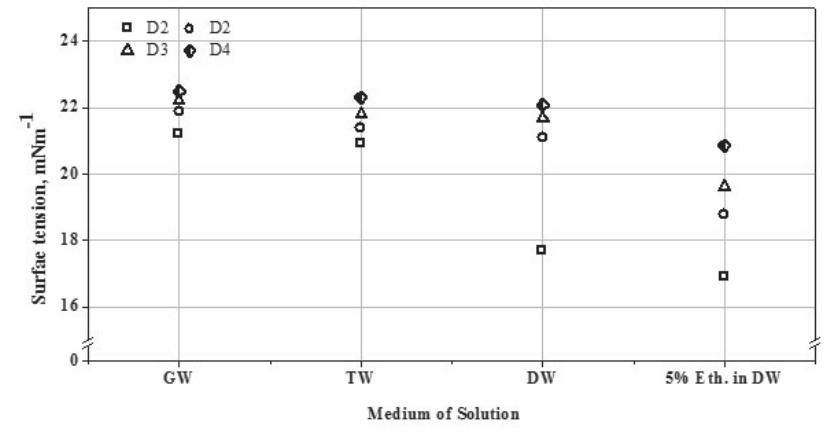

Fig. 2. Surface tension of $0.1 \% \mathrm{w} / \mathrm{v}$ of the detergent solution as a function of medium of solution

\section{$3.2 \mathrm{pH}$ of Detergent Solutions}

The $\mathrm{pH}$ of the detergent solutions D1, D2, D3 and D4 
in different medium measured at $25{ }^{\circ} \mathrm{C}$ are shown in the following Figure 3:

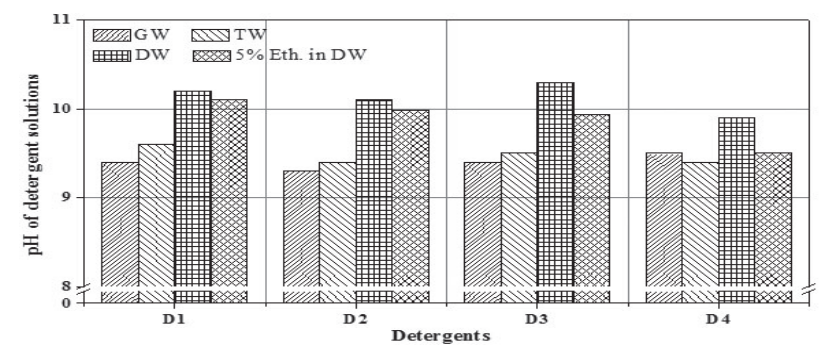

Fig. 3. $\mathrm{pH}$ of $0.1 \% \mathrm{w} / \mathrm{v}$ of detergent solution in different a medium

The $\mathrm{pH}$ of the detergent solution D1, D2, D3 and D4 in ground water (G.W.), tap water (T.W.), distilled water (D.W.) and 5\% ethanol in distilled water were found to be $9.4 \pm 0.1,9.5 \pm 0.1,10.1 \pm 0.2$ and $9.8 \pm 0.3$ respectively.

The $\mathrm{pH}$ of ground water (G.W.), tap water (T.W.) and distilled water (D.W.) were measured and found to be equal to $7.8,7.3$ and 6.3 respectively. The variation of detergent's $\mathrm{pH}$ in different mediums may be attributed to the impurities present and depend on the water source. A marked increase in $\mathrm{pH}$ was observed in the case of distilled water. The detergent's alkaline nature makes them slippery and helps the dirt particles to form micelles, which increases the cleansing action of the detergent (Tsujii 1998; Bajpai \& Tyagi 2007). Alkalinity is useful in removing acidic, fatty and oily soil; therefore, detergents are more effective in alkaline medium (www.cleaning101.com).

\subsection{Critical Micelle Concentration (CMC)}

The CMC values of the detergents D1, D2, D3 and D4 in ground water, tap water and distilled water were determined by measuring the surface tension of 0.1 to $1.0 \%$ detergent solution. At CMC, the sharp changes in surface tension value were observed and were recorded shown in the following Figure 4:

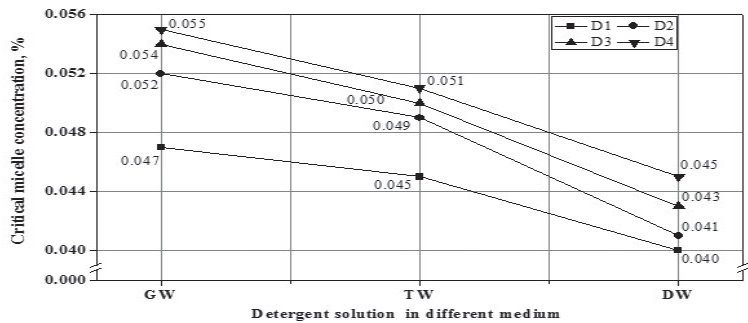

Fig. 4. CMC values of the detergents D1, D2, D3 and D4 in ground water, tap water and distilled water

The plot shows that the CMC value of the detergents decreases as D4 $>$ D3 $>$ D2 $>$ D1, also CMC values in different medium decreases as Ground water $>$ Tap water
$>$ Distilled water. The lower value of surface tension and $\mathrm{CMC}$ in distilled water, tap water and ground water require less detergent to form micelles. Therefore, the detergent's cleansing action has the following order: D1 $>$ D2 $>$ D3 $>$ D4. Also, the detergent's cleansing actions indifferent medium were found to be of the following order: Distilled water $>$ Tap water $>$ Ground water.

The CMC values largely depend on temperature, $\mathrm{pH}$, ionic strength, detergent homogeneity, purity and medium. Detergent form associative colloids in an aqueous solution that decreases water's surface tension (Hattiangdi et al. 1949; Hazra 2015). As the surface tension decreases, the extent of the adsorption of dirt in micelles increases, thereby increasing the detergent's cleansing action. The result shows that the cleansing action depend on the types and sources of water.

\subsection{Foaming Stability Test}

The foam collapsing time of the detergents D1, D2, D3 and D4 in different medium are shown in the following Figure 5:

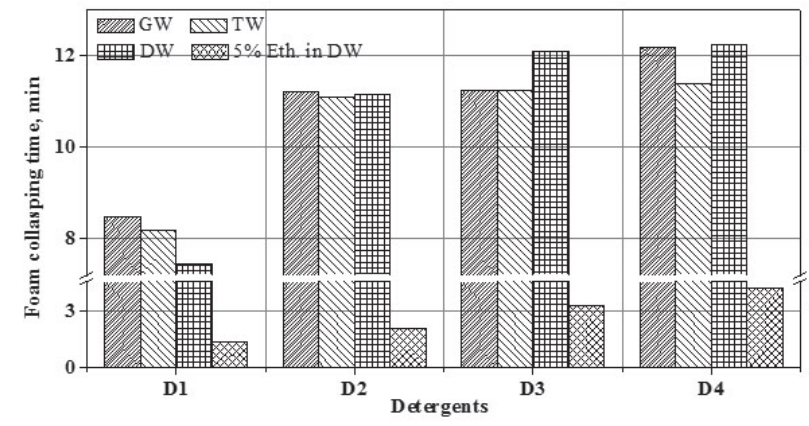

Fig. 5. Foam $(2 \mathrm{~mm})$ collapsing time of $0.1 \% \mathrm{w} / \mathrm{v}$ of detergent solution

The foam collapsing time of the detergents D1, D2, D3 and D4 in all medium, i.e., ground water (G.W.), tap water (T.W.), distilled water (D.W.) and 5\% ethanol in distilled water were found to be of following order: D1 $<$ D2 $<$ D3 $<$ D4. Also, the foam collapsing time of the detergents depends on the medium and have the following order:ground water (G.W.) $>$ tap water (T.W.) $>$ distilled water (D.W.) $>5 \%$ ethanol in distilled water. Therefore the cleansing action of the detergents were found to be of following order: D1 $>$ D2 $>$ D3 $>$ D4. And cleansing action of the detergent in different medium were found to be of following order: $5 \%$ ethanol in distilled water $>$ distilled water (D.W.) > tap water (T.W.) > ground water (G.W.).

The foam formation in dilute detergent solution shows the stabilizing action of adsorption layers which attains its 
maximum at surface concentrations and collapsing easily in case of good detergent due to the decrease of surface tension determines the cleansing action of the detergents. Also with a ethanol's Addition detergent's foam collasping time is reduced, showing better cleansing of detergent (Khetrapal et al. 2015).

\subsection{Hard Water Test}

The hardness of water is mainly due to the presence of salts of calcium $(\mathrm{Ca})$ and magnesium $(\mathrm{Mg})$, iron $(\mathrm{Fe})$, manganese $(\mathrm{Mn})$ etc. The mineral salts react with soap to form an insoluble precipitate known as scum. The effectiveness of detergent is reduced due to hard water. A good detergent forms less or no scum and therefore can be used effectively in hard water. The weight of scum that formed by the Addition of 10 drops of $5 \% \mathrm{CaCl}_{2}$ in the detergent solutions D1, D2, D3 and D4 are plotted in the following Figure 6:

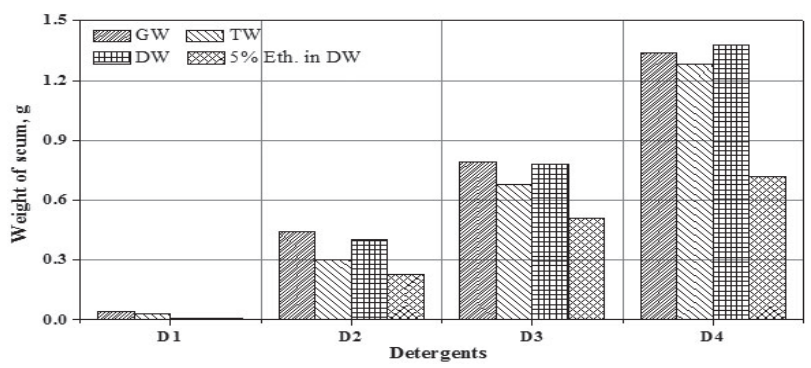

Fig. 6. Weight of scum formed after addition of $5 \% \mathrm{CaCl}_{2}$ solution in $2 \% \mathrm{w} / \mathrm{v}$ detergent solution

The minimum precipitate formation was observed in detergent D1, the lesser the weight of precipitate, the higher its cleansing action. The plot shows that the detergent's effectiveness was of the following order: D1 $>$ D2 $>$ D3 $>$ D4.

The weight of scum that formed by the Addition of 10 drops of $5 \% \mathrm{MgCl}_{2}$ in the detergent solutions D1, D2, D3 and D4 are plotted in the following Figure 7:

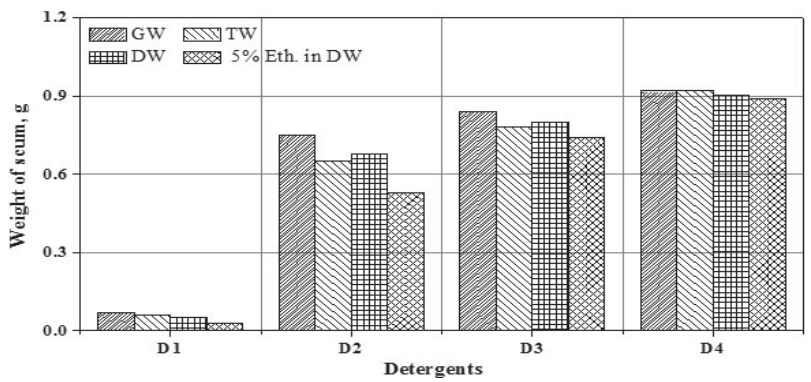

Fig. 7. Weight of scum formed after addition of $5 \% \mathrm{MgCl}_{2}$ solution in $2 \% \mathrm{w} / \mathrm{v}$ detergent solution

The plot shows that ease of precipitate formation as: D4 > D3 $>$ D2 $>$ D1. Therefore, cleansing action of detergents has the following order: D1 > D2 > D3 $>$ D4.

The weight of scum that formed by the Addition of 10 drops of $5 \% \mathrm{FeCl}_{3}$ in the detergent solutions D1, D2, D3 and D4 are plotted in the following Figure 8:

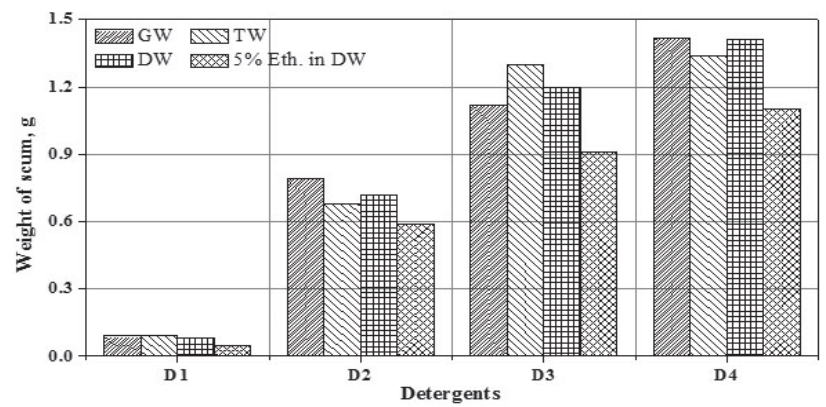

Fig. 8. Weight of scum formed after addition of $5 \% \mathrm{FeCl}_{3}$ solution in $2 \% \mathrm{w} / \mathrm{v}$ detergent solution

The minimum precipitate formation was observed in detergent D1, the lesser the weight of precipitation, the higher is cleansing action (Khetrapal et al. 2015). Generally Softeners such as sodium tripolyphosphate are used to soften hard water. In case of the detergent solution D1, after the addition of $\mathrm{CaCl}_{2}, \mathrm{MgCl}_{2}$ and $\mathrm{FeCl}_{3}$, the order of scum formation is $\mathrm{GW}>\mathrm{TW}>\mathrm{DW} \geq 5 \%$ Ethanol in DW, mainly it may be due to the presence of soluble salts of calcium and magnesium ions. But in case of the detergent samples, D2, D3 and D4, scum formation is slightly higher in distilled water; it may be due to experimental indistinguishable weighing differences and the presence of poor quality of softener in the detergents.

\subsection{Emulsion Stability Test}

The emulsion stability of detergents D1, D2, D3 and D4, when $1 \%$ solution of detergent and $0.5 \mathrm{~mL}$ of mustard oil was shaken for 1 minute, are plotted in the following Figure 9:

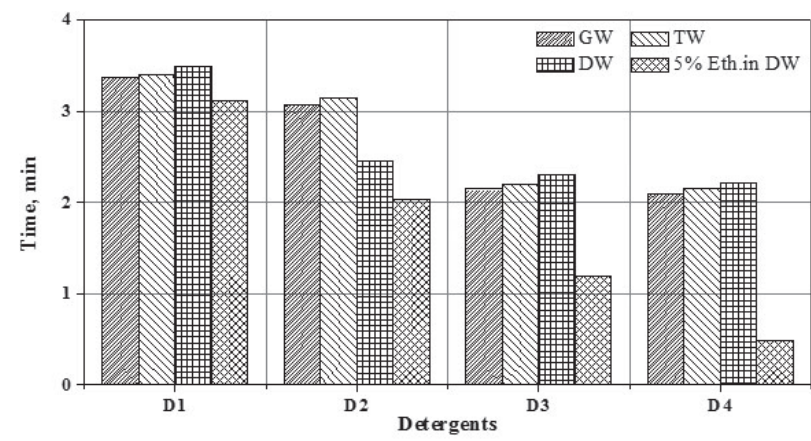

Fig. 9. Emulsification of $1 \% \mathrm{w} / \mathrm{v}$ of detergent solutions on addition of $0.5 \mathrm{~mL}$ mustard oil

Emulsion formation is the basis of cleansing action. On increasing detergent concentration, emulsion's stability 
also increases and the time required for separating the layers increases. The more significant time required for separating the layers, the greater is the detergent's cleansing action (Kamba et al. 2013). In case of 5\% ethanol in water, the emulsion gets quickly stabilized compared to ground, tap and distilled water. It may be due to the decrease in the emulsion's droplet size, contributing to an emulsion's stability and esterification of isothiocyanates group present in mustard oil in presence of ethanol.

The emulsion stability of detergents D1, D2, D3 and D4, when $1 \%$ solution of detergent and $0.5 \mathrm{~mL}$ of petrol was shaken for 1 minute, are plotted in the following Figure 10:

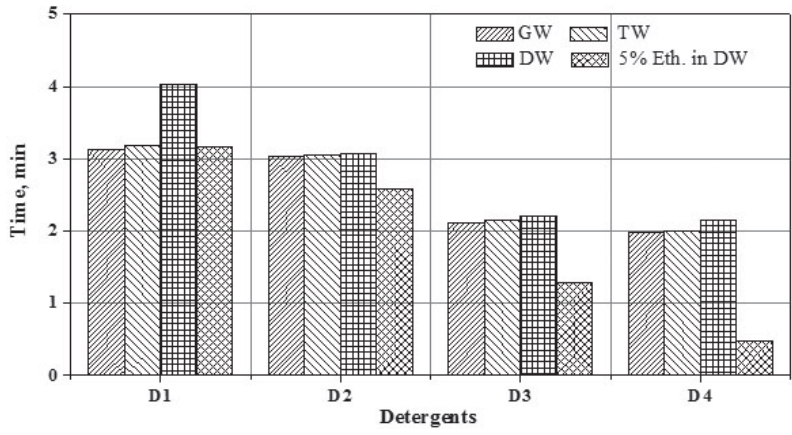

Fig. 10. Emulsification of $1 \% \mathrm{w} / \mathrm{v}$ of detergent solutions on addition of $0.5 \mathrm{~mL}$ petrol

The detergents' emulsion stability tests in all medium were of the following order: D1 > D2 > D3 $>$ D4. At low surfactant concentration, micro-emulsions are formed due to ultralow oil-water interfacial tension. The hydrophobic and hydrophilic parts of the surfactants allow adsorbing to non-polar and polar materials simultaneously. More significant the emulsion stability lower is the extent of surface tension thereby greater is the cleansing action of the detergents (Bajpai \& Tyagi 2007; Kamba et al. 2013)

\section{CONCLUSION}

The detergent's physical properties such as surface tension, $\mathrm{pH}$, critical micelle concentration, foam stability, the hardness of water, and emulsion stability were measured, and their cleansing actions in the different aqueous medium were compared. The detergent's surface tension was found to be of the following order: D1 $<$ D2 $<$ D $3<$ D4 . The critical micelle concentration of detergents D1, D2, D3 and D4 in ground water (G.W.), tap water (T.W.) and distilled water (D.W.) are found as D1 $<$ D2 $<$ D3 $<$ D4. All detergents' $\mathrm{pH}$ was found alkaline at room temperature, and detergent D1 was found most alkaline. From the foam collapsing time of the detergents, hard water test, i.e., scum formations, emulsion stability test of detergents, the detergents' cleansing action has detergents' cleansing action has shown in the following order: D1 $>$ D2 $>$ D3
$>$ D4. Also, the cleansing action of the detergents in the different aqueous medium has shown the following order: $5 \%$ ethanol in distilled water $>$ distilled water (D.W.) $>$ tap water (T.W.) $>$ ground water (G.W.).

Among the four detergents, the surface tension and $\mathrm{CMC}$ value of the detergent D1 were found least among the four detergents; the less CMC value shows that less detergent is required to form micelles therefore, is of good quality. The foam collasping time of the detergents D1 was least; it is due to the decrease of surface tension this determines the cleansing action of suitable detergents. The weight of the scum formed was least when the detergent D1 was treated with hared water. Emulsion stability of the detergent D1 in all medium was found maximum. These results show that the detergent D1 has superior cleansing action in all medium. However, medium of detergent solution plays an important role on its cleansing action.

From this study it is recommended that detergents available in Nepalese market may be categorized into different grades on the basis of cleansing action specifying the medium and is expected to provide a guideline for consumer in the selection of detergent.

\section{ACKNOWLEDGEMENT}

The authors are grateful to the Head of the Department of Chemistry, Tri-Chandra Multiple Campus, for providing the physical facilities to carry out this study.

\section{REFERENCES}

1. Bajpai, D. and V.K. Tyagi. 2007. Laundary Detergents: An Overview, Journal of Oleo Science, 56(7): 327-340.

2. Bratovcic, A., S. Nazdrajic, A. Odobasic and I. Sestan. 2018. The Influence of Type of Surfactant on Physicochemical Properties of Liquid Soap, International Journal of Materials and Chemistry, 8(2): 31-37.

3. Chakraborty, T., I. Chakraborty and S. Ghosh. 2011. The methods of determination of critical micellar concentrations of the amphiphilic systems in aqueous medium, Arabian Journal of Chemistry, 4: 265-270

4. Culter, W.G. 1972. Detergency: Part-1, Surfactant Science Series, Marcel Dekker, 9-15.

5. Elarbi, F.M., A.A. Janger, L.M. Abu-sen and Z.O. Ettarhouni. 2020. Determination of $\mathrm{CMC}$ and interfacial properties of anionic (SDS) and cationic (CPB) surfactants in aqueous solutions, American Journal of Engineering Research, 9(8): 118-126. 
6. Hattiangdi, G.S., W.W. Walton and J. Hoffman. 1948. Some Physical Chemical Properties of Aqueous Solutions of Soaps and Soapless Detergents, Journal of Research of the National Bureau of Standards, 42: 361-368.

7. Hazra, A. 2015.Comparative Study of Some House-Hold Surface Active Substances, International Journal of Scientific Research in Science and Technology, 1(3): 75-79.

8. Kamba, E.A., A.U. Itodo and E. Ogah. 2013. Utilization of Different Emulsifying Agents in the Preparation and Stabilization of Emulsions, International Journal of Materials and Chemistry, 3(4): 69-74.

9. Khan, A. and S. Shah. 2008. Determination of critical micelle concentration (CMC) of sodium dodecyl sulfate (SDS) and the effect of low concentration of pyrene on its CMC using Origin, Journal of Chemistry, 2: 46-186.

10. Khetrapal, M., P. Mudgal, V. Lata, Sagarika, Ayushi, Vishu, Vaishali, Ushma, Deepika and Charu. 2015. Comparative Study of Detergents in India-A Step towards More Sustainable Laundry, DU Journal of Undergraduate Research and Innovation, 163-172.

11. Limbu, K., S.K. Shah and A. Bhattarai. 2014. Micellization behaviour of sodium dodecyl sulphate in presence and absence of sodium sulphate and zinc sulphate in distilled water by surface tension measurement, Bibechana, 11(1): $79-85$.
12. Pisarcik, M., F. Devinsky and P. Matus. 2015. Determination of micelle aggregation numbers of alkyltrimethylammonium bromide and sodium dodecyl sulfate surfactants using timeresolved fluorescence quenching, Open Chemistry, 13: 922-931.

13. Saha, D., M. Hait, M. Patanwar and A. Tamarakar. 2011. Studies on surface tension of selected juice formulation by drop number method using Traube's stalegmometer technique. Bulletin of Pharmaceutical Research, 1(3): 1-3.

14. Soap and Detergents, The soap and Detergent Association. 1994. $2^{\text {nd }}$ edition, Washington DC, 4-33. (www.cleaning101. com)

15. Tsujii, K., 1998. Surface activity: Principles, Phenomena and Applications, Academic Press, San Diego, USA, 182-201.

16. Taylor, T.P., K.M. Rathfelder, K.D. Pennell and L.M. Abriola. 2004. Effects of ethanol addition on micellar solubilization and plume migration during surfactant enhanced recovery of tetrachloroethene. Journal of Contaminant Hydrology, 69(2):73-99.

17. Vazquez, G., E. Alvarez and J.M. Navaza. 1995. Surface Tension of Alcohol + Water from 20 to $50{ }^{\circ} \mathrm{C}$, Journal of Chemical and Engineering Data, 40: 611-614. 